\title{
Developing Transdermal Applications of Ketorolac Tromethamine Entrapped in Stimuli Sensitive Block Copolymer Hydrogels.
}

M. Mallandricha,f, F. Fernández- Camposa, L. Halbauta, C. Alonsob/L. Coderchb, M.L. Garduño-Ramírezc / B. Andradec, A. del Pozo a, M. Laned, B. Clarese, A.C. Calpena ${ }^{\mathrm{a}, \mathrm{f}}$

\footnotetext{
a Department of Pharmacy, Pharmaceutical Technology and Physical Chemistry. Faculty of Pharmacy. University of Barcelona (UB), Joan XXIII Avenue, 27-31. Barcelona 08028. Spain.

${ }^{\mathrm{b}}$ Institute of Advanced Chemistry of Catalonia. Jordi Girona Street, 18-26. Barcelona 08034. Spain.

${ }^{\text {c }}$ Centro de Investigaciones Químicas, Universidad Autónoma del Estado de Morelos. Avenida Universidad 1001, Cuernavaca 62209, Morelos. Mexico.

${ }^{\mathrm{d}}$ UCL School of Pharmacy, 29-39 Brunswick Square, London, WC1N 1AX, United Kingdom

e Department of Pharmacy and Pharmaceutical Technology. Faculty of Pharmacy. University of Granada. Campus of Cartuja s/n, Granada 18071. Spain.

${ }_{\mathrm{f}}^{\mathrm{f}}$ Nanoscience and Nanotechnology Institute (IN2UB). University of Barcelona (UB), Joan XXIII Avenue, 27-31. Barcelona 08028. Spain.
} 


\section{ABSTRACT}

PURPOSE:

In order to obtain dermal vehicles of ketorolac tromethamine (KT) for the local treatment of inflammation and restrict undesirable side effects of systemic levels hydrogels (HGs) of poloxamer and carbomer were developed.

\section{METHODS:}

KT poloxamer based HG (KT-P407-HG) and KT carbomer based HG (KT-C940-HG) were elaborated and characterized in terms of swelling, degradation, porosity, rheology, stability, in vitro release, ex vivo permeation and distribution skin layers. Finally, in vivo anti-inflammatory efficacy and skin tolerance were also assessed.

RESULTS:

HGs were transparent and kept stable after 3 months exhibiting biocompatible near neutral pH values. Rheological patterns fitted to Herschel-Bulkley for KT-C940-HG and Newton for KT-P407-HG due to its low viscosity at $25^{\circ} \mathrm{C}$. Rapid release profiles were observed through first order kinetics. Following the surface the highest concentration of KT from C940-HG was found in the epidermis and the stratum corneum for P407HG. Relevant anti-inflammatory efficacy of KT-P407-HG revealed enough ability to provide sufficient bioavailability KT to reach easily the site of action. The application of developed formulations in volunteers did not induce any visual skin irritation.

CONCLUSIONS:

KT-P407-HG was proposed as suitable formulation for anti-inflammatory local treatment without theoretical systemic side effect.

\section{KEYWORDS:}

carbomer; hydrogels; ketorolac tromethamine; poloxamer; transdermal 


\section{Introduction}

Ketorolac tromethamine (KT) belongs to the pyrrolo-pyrrole group of nonsteroidal anti-inflammatory drugs with potent analgesic and moderate anti-inflammatory activities used for the short-term management of moderate to severe acute postoperative pain $(1,2)$. At present this drug is administered either intramuscularly or orally for the short-term management of post-operative pain and as an ophthalmic suspension for the prophylaxis and reduction of postoperative ocular inflammation. Injections, however, are invasive and often inconvenient for patients, especially in terms of self-administration. Oral therapy requires a frequent dosing regimen due to a biological halflife of 4-6 h with associated adverse effects such as, peptic ulceration and gastrointestinal bleeding.

The application of this type of drugs via the dermal route could offer additional advantages, e.g. it avoids the first pass metabolism, averts the risk of gastrointestinal disturbance, targeting only the areas of disease (3). However, the stratum corneum (SC) of the skin represents a formidable barrier which is the rate-limiting step for permeation across the skin (4). To overcome this problem, and to enhance drug bioavailability, the active is incorporated into different drug delivery systems based on polymeric nanostructures (5). Among these drug delivery systems, hydrogels emerged as the third generation biomaterial systems that function as drug delivery systems (6). Hydrogels (HGs) are three-dimensional cross-linked polymeric networks that exhibit the ability to swell and retain a large amount of water, without dissolution (7). Their highly porous structure can easily be tuned by controlling the density of cross-links in the gel matrix and the affinity of the HG for the aqueous environment in which they are swollen $(8,9)$. By modifying the HG density, the rate at which an entrapped drug is released can be altered to allow the delivery of the drug over a specific period (10). Concretely, micro/ nanostructured polymeric systems have attracted much attention because of their pharmaceutical applications. In particular, poloxamer, also known as pluronic $\AA$, is a amphiphilic thermoresponsive block copolymer consisting of a central hydrophobic block of Poly(propylene oxide) (PPO) flanked by hydrophilic Poly(ethylene oxide) (PEO) blocks (PEO-PPO- PEO), has held interest in the design of dermal and transdermal delivery systems with a view to promoting, improving or retarding drug permeation through the skin (11).

On the other hand, carbomer, also known as carbopol ${ }^{\circledR}$, is a hydrophilic $\mathrm{pH}$ responsive acrylic acid polymer cross-linked with polyalkenyl ethers or divinyl glycol (12), with optimal topical applications (13). In this study is evaluated if poloxamer and carbomer HGs can be used to produce dermal vehicles of KT for the local treatment of inflammation without reaching systemic levels in order to restrict undesirable side effects of KT. For that, this work aimed at developing KT delivery systems with improved biopharmaceutical profile for dermal administration. Thus, to target KT in the skin in a controlled release manner enhancing the contact of KT with the skin and improving its retention, we elaborated a poloxamer based HG and a carbomer based HG loading KT. After physical characterization in terms of rheological behavior and stability, in vitro release, as well as, ex vivo permeation studies of KT from HGs were accomplished. Finally, in vivo skin tolerance and antiinflammatory efficacy were also assessed.

\section{Materials and methods}

\subsection{Materials}

Ketorolac tromethamine was purchased from Sigma-Aldrich (Barcelona, Spain). Carbopol® 940 and Pluronic ${ }^{\circledR}$ F-127 were obtained from Fagron Iberica (Terrassa, Spain). Sodium phosphate dibasic $\left(\mathrm{Na}_{2} \mathrm{HPO}_{4}\right)$ and monopotassium phosphate $\left(\mathrm{KH}_{2} \mathrm{PO}_{4}\right)$ were supplied by Panreac (Barcelona, Spain). The purified water used in all the experiments was obtained from a Milli-Q1 Gradinet A10 system apparatus -home supplied- (Millipore Iberica S.A.U., Madrid, Spain). All the other chemicals and reagents used in the study were of analytical grade.

Tissues for ex Vivo Assays Ear porcine (Landrace Large White race) skin was obtained from the Bellvitge animal facility services, and approved by the Ethics Committee of Animal Experimentation of the University of Barcelona. $1 \mathrm{~mm}$ thickness skin tissue was used for experiments. The skin was initially cleaned with tap water, and then hairs and subcutaneous fat tissue were removed with a scalpel. Human skin was obtained from the abdominal region of healthy women (plastic surgery department, BarcelonaSCIAS Hospital, Barcelona, Spain). The experimental protocol was approved by the Bioethics Committee of the Barcelona-SCIAS Hospital and written informed 
consent forms were provided by volunteers. After being frozen to $-20^{\circ} \mathrm{C}$, tissues were dermatomed (GA630, Aesculap, Tuttlingen, Germany) into $500 \mu \mathrm{m}$-thick pieces. Human skin integrity was verified by measuring the trans epidermal water loss (TEWL) using a TEWL-meter TM210 (Courage \& Khazaka, Koln, Germany). Human skin pieces exhibiting TEWL values above $10 \mathrm{~g} / \mathrm{m} 2 \mathrm{~h}$ were ruled out. Experimental Animals Male Swiss CD-1 mice (20-25 g) were acquired from Círculo ADN S.A. de C.V. (Coyoacan D.F., Mexico) and were subjected to a quarantine period of 7 days on arrival. The animals were housed in plastic cages with soft bedding with access to controlled diet and tap water ad libitum. The temperature was kept at $24 \pm 1^{\circ} \mathrm{C}$ and the relative humidity was kept at 50-60\%. Artificial lighting was used to provide $12 \mathrm{~h}$ light and $12 \mathrm{~h}$ dark every $24 \mathrm{~h}$. The studies were conducted under a protocol in accordance with the Mexican Official Normative for Animal Care and Handling (NOM-062ZOO-1999) and with the approval of the Academic Committee of Ethics of the Vivarium at the Universidad Autónoma del Estado de Morelos (Mexico).

\section{Elaboration of $H G s$}

P407 and C940 were used as polymers for the preparation of two HGs (18\% and 2\%, respectively). KT loaded HGs (KTP407-HG and KT-C940-HG) were elaborated at laboratory scale at a concentration of 2\% (w/v) as previously described (11). Briefly, KT was dissolved in distilled water. The preweighed quantity of polymer was gradually added to this solution under continuous stirring, until a thin dispersion, without residual powder, was formed. HGs were then kept in a tightly closed container at the following conditions: P407 was kept at $4^{\circ} \mathrm{C}$ for 24 $\mathrm{h}$ and C940 was allowed to swell for $24 \mathrm{~h}$ at room temperature, and then, triethanolamine was added to the formulation. Once elaborated, both formulations were stored at room temperature until following studies.

\section{Physical Characterization}

All HGs formulations were visually observed immediately and 3 months after preparation for color, odor and viscosity. The $\mathrm{pH}$ of the prepared $\mathrm{HGs}$ was measured at room temperature and $32^{\circ} \mathrm{C}$, using the CRISON micro-pH 200 microprocessor controlled pH-meter (Crison Instruments S.A., Barcelona, Spain), immediately after preparation of formulations and after three months.

\section{Swelling and Degradation Tests}

The swelling ratio (SR) was assessed by a gravimetric method. Briefly, dried HGs were incubated in PBS (pH $=5.5$ ) at $32^{\circ} \mathrm{C}$ for $24 \mathrm{~h}$ in the case of KT-C940-HG and $30 \mathrm{~min}$ KT-P407- HG. At predetermined times; samples were removed and weighed $(\mathrm{Wt})$ after blotting the surface water. The PBS uptake was carried out in triplicate. The swelling ratio was calculated based on the following equation:

$$
\mathrm{QR}(\%)=\mathrm{Ws}-\mathrm{Wd} / \mathrm{Wd}
$$

Where Ws is the weight of the swollen HG at different times and Wd is the weight of dried HG. The degradation as percentage of weight loss (WL) was calculated by immersing known amounts of dried HGs in $\mathrm{PBS}$ ( $\mathrm{pH}=$ 5.5) at $32^{\circ} \mathrm{C}$ for $24 \mathrm{~h}$ in the case of KT-C940- HG and $19 \mathrm{~min}$ for KT-P407-HG. At regular time intervals, samples $(\mathrm{n}=3)$ were dried and weighed. WL was calculated according to the equation:

$$
\mathrm{WL}(\%)=(\mathrm{Wi}-\mathrm{Wd} / \mathrm{Wi}) \times 100
$$

Where $\mathrm{Wi}$ is the initial weight of $\mathrm{HG}$ and $\mathrm{Wd}$ the weight of $\mathrm{HG}$ at different times.

\section{Porosity and Morphological Studies}

The porosity (P) was estimated by the solvent replacement method, which consisted in submerging the dried HG in absolute ethanol for $4 \mathrm{~h}$ in the case of KT-C940-HG and 2 min for KT-P407-HG, and then weighed after the excess ethanol on the surface was blotted. The porosity was calculated according to the equation:

$$
\mathrm{P}=(\mathrm{W} 2-\mathrm{W} 1 / \rho \times \mathrm{V}) \times 100
$$


Where W1 stands for the weight of the dried HG to be immersed in ethanol, W2 represents the weight of HG after being immersed in ethanol, $\rho$ and $\mathrm{V}$ are the density of absolute ethanol and the volume of the hydrogel, respectively. Furthermore, the structure of HGs was examined by Scanning Electron Microscopy (SEM) in a JEOL J-7100F (JEOL Inc., Peabody, MA, USA) by coating with a thin layer of carbon in an Emitech K950X coater (Quorum Technologies Ltd., Kent, UK).

\section{Analytical Conditions and KT Content}

The amount of KT in samples was determined by high performance liquid chromatography (HPLC) methodology validated according ICH Q2 (R1) validation guidelines in terms of linearity, accuracy and precision (14). HPLC system consisted of Hitachi-Merck HPLC equipment with a variable L-4250 UVVis detector $(\lambda=314 \mathrm{~nm})$, and a C18 column (LiChrocart 250-4/LiChrosorb RP-18, $5 \mu \mathrm{m}$ ) with a flow rate of $1 \mathrm{~mL} / \mathrm{min}$ at isocratic conditions. The injection volume was $20 \mu \mathrm{L}$. Elution conditions for detection of KT were sodium dihydrogen phosphate (pH 2.9) (Sigma, St Louis, MO, USA) /methanol (Merck, Darmstadt, Germany) (450/550, v/v) at $314 \mathrm{~nm}$. The KT content in HGs was addressed as follows: an exact volume of $10 \mu \mathrm{L}$ of each formulation was dissolved in $10 \mathrm{~mL}$ of water:methanol (1:1) under stirring for $15 \mathrm{~min}$ in an Elma Transsonic Digital S T490 DH ultrasonic bath (Elma, Singen, Germany). Solutions were filtered through a $0.45 \mu \mathrm{m}$ Cameo ${ }^{\circledR}$ syringe filter nylon membrane (Sigma Aldrich, Barcelona, Spain) and analyzed by HPLC.

\section{Rheological Study}

Rheological properties were determined using a rotational Haake RheoStress 1 rheometer (Thermo Fisher Scientific, Karlsruhe, Germany) equipped with cone-plate geometry (Haake C60/2 ${ }^{\circ} \mathrm{Ti}, 60 \mathrm{~mm}$ diameter, $0.105 \mathrm{~mm}$ gap between cone-plate) at 25 and $32^{\circ} \mathrm{C}$ (Thermo Haake Phoenix II + Haake C25P). Samples underwent a program consisting of 3 steps shear profile; firstly, a ramp-up period from 0 to $100 \mathrm{~s}-1$ during 3 min, followed by a constant shear rate period at $100 \mathrm{~s}^{-1}$ for $1 \mathrm{~min}$ and finally the ramp-down period from 100 to $0 \mathrm{~s}-1$ for $3 \mathrm{~min}$. Steady-state viscosity determined at t0 $\left(25\right.$ and $\left.32^{\circ} \mathrm{C}\right)$, was also calculated from the constant shear stretch at 100 $\mathrm{s}-1$. The gelation temperature of KT-P407-HG was also evaluated according to Brugués et al. (11). Briefly, the formulation was placed in a water bath under stirring with a magnetic bar. The temperature of the hydrogel was steadily increased from 21 to $36^{\circ} \mathrm{C}$. The gelation temperature was assumed as the one that the magnetic bar stopped because of gelation. The experiment was carried out by triplicate.

Data Analysis

The obtained flow data of KT-P407-HG and KT-C940-HG were fitted to different mathematical models in order to characterize flow properties and to describe the data:

\begin{tabular}{|l|c|}
\hline Newton & $\tau=\eta \cdot \dot{\gamma}$ \\
\hline Bingham & $\tau=\tau_{0}+\left(\eta_{\mathrm{p}} \cdot \dot{\gamma}\right)$ \\
\hline Ostwald-de-Waele & $\tau=\mathrm{K} \cdot \dot{\gamma}^{\mathrm{n}}$ \\
\hline Herschel-Bulkley & $\tau=\tau_{0}+\mathrm{K} \cdot \dot{\gamma}^{\mathrm{n}}$ \\
\hline Casson & $\tau=\sqrt[n]{\left(\tau_{0}^{\mathrm{n}}+\left(\eta_{0} \cdot \dot{\gamma}\right)^{\mathrm{n}}\right)}$ \\
\hline Cross & $\tau=\dot{\gamma} \cdot\left(\eta_{\infty}+\left(\eta_{0}-\eta_{\infty}\right) /\left(1+\left(\dot{\gamma} / \dot{\gamma}_{0}\right)^{\mathrm{n}}\right)\right.$ \\
\hline
\end{tabular}

Where, $\tau$ is the shear stress $(\mathrm{Pa}), \gamma$ is the shear rate $(1 / \mathrm{s}), \eta$ is the dynamic viscosity $(\mathrm{mPa} \cdot \mathrm{s}), \tau 0$ is the yield shear stress $(\mathrm{Pa}), \eta 0$ is the zero shear rate viscosity, $\eta p$ is a constant plastic viscosity $(\mathrm{mPa} \cdot \mathrm{s}), \eta \infty$ is the infinity shear rate viscosity, $\mathrm{n}$ is the flow index and $\mathrm{K}$ is the consistency index. Best fitting was based on the calculation of the correlation coefficient ( $\mathrm{r}$ ) by linear regression analysis of the flow plots.

\section{In Vitro Release}


The release assay was performed using thermostated $\left(32 \pm 0.5^{\circ} \mathrm{C}\right)$ amber glass Franz-type diffusion cells (FDC 400, Crown Glass, Somerville, NY, USA) with an active diffusion area of $2.54 \mathrm{~cm}^{2}$, cellulose dialysis membranes with molecular weight cut-off of 12KDa (Iberlavo, Madrid, Spain), and phosphate buffer solution (PBS) (0.06 M, $\mathrm{pH}$ 7.6) under continuous stirring as receptor medium, assuring sink conditions. $100 \mathrm{mg}$ samples were placed in the donor compartment and sealed with Parafilm ${ }^{\circledR}$ to avoid evaporation. $300 \mu \mathrm{L}$ samples were collected via syringe at pre-stablished intervals for $9 \mathrm{~h}$, and immediately replaced with similar volume and temperature of PBS. The collected samples were analyzed for drug content by HPLC. The experiment was performed in quadruplicate, and the cumulative percentage of KT released was calculated.

\section{Kinetics}

The amount of KT released was fitted to the following model equations:

$\begin{array}{ll}\text { Zero-order } & \% R t / \% R \infty=K o \times t \\ \text { First-order } & \% R t / \% R \infty=\left(1-e^{k \times t}\right) \\ \text { Higuchi } & \% R t / \% R \infty=K h \times t^{1 / 2} \\ \text { Korsmeyer-Peppas } & \% R t / \% R \infty=K \times t^{n}\end{array}$

Where Rt is the amount of drug released at time (t), $\mathrm{R} \infty$ is the maximum amount of drug released, $\mathrm{k}$ is the release rate constant expressed in units of concentration/time, and $\mathrm{n}$ is the diffusion release exponent that can be used to characterize the different release mechanisms. It has been established that $\mathrm{n} \leq 0.43$ (Fickian diffusion mechanism), $0.43<\mathrm{n}<0.85$ (anomalous transport) and $\mathrm{n} \geq 0.85$ (super case II transport, i.e. zero, order release). A nonlinear least squares regression was performed using the WinNonLin ${ }^{\circledR}$, V. 3.3 software (Pharsight Co., MountainView, CA, USA). The Akaike information criterion (AIC) was determined for each model as an indicator of the model's suitability for a given dataset based on maximum likelihood.

\section{Ex Vivo Permeation Protocols}

The following guidelines were adhered during these studies: OECD guidelines $(15,16)$, the published opinions of the Scientific Committee on Cosmetic Products and Non-Food Products (17) and classical and updated principles of percutaneous absorption (18). Estimation of Permeation Parameters The estimation of permeation parameters was carried out using Franz diffusion cells as described previously. The ear pig skin was mounted between the receptor and donor compartments with the SC towards the donor chamber with a diffusion area of $2.54 \mathrm{~cm}^{2} .300$ mg samples were placed in the donor compartment in contact with porcine skin. Samples of the receptor phase (300 $\mu \mathrm{L}$ aliquots) were withdrawn periodically and replaced by receptor medium at various time intervals for $24 \mathrm{~h}$, and analysed by HPLC for drug content. Six parallel determinations were addressed. Once the experiment finalized, the amount of drug retained (AR, $\mu \mathrm{g} / \mathrm{g}$ skin $/ \mathrm{cm}^{2}$ ), in the porcine membrane was determined. For that purpose, the skin was cleaned with sodium lauryl sulphate solution $0.05 \%$ and rinsed with distilled water. The permeation area was excised and weighed, then the drug reatined was extracted with methanol:water (50:50, v:v) under sonication for $20 \mathrm{~min}$ in ultrasound bath, and analysed by HPLC.

The following parameters were calculated from experimental data:

The cumulative amount of KT permeated through porcine skin membrane was plotted as a function of time. The slope and intercept of the linear portion of the plot was derived by regression using the Prism®, V. 5 software (GraphPad Software Inc., San Diego, CA, USA). KT fluxes $\left(\mathrm{J}, \mu \mathrm{g} / \mathrm{cm}^{2} / \mathrm{h}\right)$ from the assayed formulations were calculated from the slope of linear portion of the cumulative amounts permeated through the membrane per unit surface area versus time plot, and the extrapolation of this line will intercept with the X-axis at a time equal to lag time $(\mathrm{TL}, \mathrm{h})$. The permeability coefficients $(\mathrm{Kp}, \mathrm{cm} / \mathrm{h})$ were obtained by dividing the $\mathrm{J}$ by the initial drug concentration $(\mathrm{C} 0)$ in the donor compartment, and it is assumed that under sink conditions the drug concentration in the receiver compartment is negligible compared to that in the donor compartment (19). The predicted steady-state 
plasma concentration (Css) of drug, which would penetrate skin barrier after topical application, was obtained using the following equation:

$\mathrm{C}_{\mathrm{ss}}=(\mathrm{J} \cdot \mathrm{A}) /\left(\mathrm{Cl}_{\mathrm{p}} \cdot \mathrm{A}\right)$

where $\mathrm{C}_{\mathrm{ss}}$ is the plasma steady-state concentration, $\mathrm{J}$ the flux determined in this study, A the hypothetical area of application and $\mathrm{Cl}_{\mathrm{p}}$ the plasmatic clearance.

\section{Percutaneous Absorption and KT Distribution in Human Skin}

Layers Human skin discs with an internal diameter of $2.5 \mathrm{~cm}$ were placed on thermostated $\left(32^{\circ} \mathrm{C}\right)$ Franz static diffusion cells ( $3 \mathrm{~mL}, 1.86 \mathrm{~cm}^{2}$ of exposed area, Lara-Spiral, Courtenon, France) in order to determine the compartmental distribution of KT after an exposure time of $24 \mathrm{~h}$. PBS at pH 7.6 (Sigma, St Louis, USA) in water (MilliQ quality) was used as receptor fluid, representing the $\mathrm{pH}$ of blood. The solubility of KT in the receptor medium and sink conditions were guaranteed throughout the assay by adding gentamicin sulphate $0.04 \%(\mathrm{w} / \mathrm{v})$ (Sigma, St Louis, MO, USA) and bovine serum albumin 1\% (w/v) (Sigma, St Louis, MO, USA) under magnetic stirring (20). Samples $(10 \mu \mathrm{L}$ ) were applied to the skin. Control cells were also used, $10 \mu \mathrm{L}$ of blank HGs (without KT) were applied to the skin surface to evaluate potential interferences of the HG or skin layer in the analysis of KT.

After $24 \mathrm{~h}$ exposure period, the skin discs were disassembled from the Franz cells and the excess of HGs formulations was removed from the skin in order to recover the excess of KT. Then, the receptor fluid was recovered, and stripping procedures were performed on the surface horny layers of the SC with adhesive tape (D-squame, Cuderm Co., Dallas, USA). Twelve strips were carried out in order to remove most of the amount of substance contained in the SC. The epidermis was separated from the dermis after heat treatment (20). The different samples to be analyzed were extracted and/or diluted in water:methanol (1:1,v/v) (Merck, Darmstadt, Germany). $10 \mathrm{~mL}$ of the extraction solvent was added to the $\mathrm{S}$ sample, $2 \mathrm{~mL}$ of extraction solvent were used for the $\mathrm{SC}$ and $\mathrm{E}$, and 1 $\mathrm{mL}$ was used for the extraction of KT from D. The resulting mixtures were sonicated for 20 min and the supernatants were pipetted and analyzed by HPLC, yielding the amount of $\mathrm{KT}$ as $\mu \mathrm{g}(\mathrm{KT}) /[\mathrm{g}($ human skin) $/ \mathrm{cm} 2]$ and $\%$ of the applied dose.

\section{In Vivo Anti-Inflammatory Efficacy}

Anti-inflammatory efficacy of developed HGs was assessed according to the protocol described by Domínguez-Villegas et al. (21). Briefly, $2.5 \mathrm{mg}$ of 12- O-detradecanoylphorbol-13-acetate (TPA) dissolved in 5 $\mu \mathrm{L}$ ethanol was used to induce mouse ear edema inflammation in mice. The animals were divided in different groups $(\mathrm{n}=3$ ). $100 \mathrm{mg}$ of KT-P407-HG (group 1) or KT-C940-HG (group 2) were applied to both sides of the right ear simultaneously with TPA, also $5 \mu \mathrm{L}$ of ethanol was applied to both sides of the left ear. An equivalent KT solution in acetone was assayed similarly as reference (group 3) to both sides of the right ear and $5 \mu \mathrm{L}$ of acetone to both sides of the left ear. $100 \mathrm{mg}$ of blank HGs (groups 4 and 5) were applied to both sides of the right ear simultaneously with TPA and without TPA in both sides of the left ear. Four hours after the application of the formulations, the animals were sacrificed by cervical dislocation and circular sections with $7 \mathrm{~mm}$ of diameter were cut from left and right ears and weighted to determine the anti-inflammatory activity expressed as the percentage of inhibition of the inflammatory process according to the equation:

Inhibition $\%=$ (difference in weight of ear; control-difference in weight of ear; treated difference in weight of ear/control) x 100

\section{Histological Evaluation}

After the anti-inflammatory efficacy test, the circular sections of ears of treated animals, as well as, positive and negative controls were histologically evaluated to confirm the antiinflammatory efficacy of HGs by detecting the presence of cells from the immune system in tissues. For this task, tissues were fixed in a $4 \%$ formaldehyde solution. Samples were embedded in paraffin and histological slices were obtained using standard procedures. Subsequently, samples were conventionally stained with hematoxylin-eosin and observed by light microscopy. 


\section{In Vivo Tolerance Study}

Ten female volunteers with healthy skin between $21-52$ years old participated in the study. The study was approved by the Ethics Committee of the University of Barcelona according to the recommendations of the Declaration of Helsinki (22), all the subjects provided signed a written informed consent forms. Subjects were also instructed for not to use skin cleansing or skin care products on the test sites during two days before and during the study. Prior to the measurements, the volunteers were allowed to stay in the test room for at least 30 min prior to the measurements.

Several measurements were performed before applying the formulation (baseline readings), immediately after application as a uniform layer $(0.1 \mathrm{~mL} / \mathrm{cm} 2)$ and one hour after application (23), on the flexor side of the left and right forearm for KT-P407-HG and KT-C940-HG, respectively. Skin temperature was measured using a Skin Thermometer® ST500 (Courage-Khazaka electronic GmbH, Cologne, Germany). TEWL, referring to the total amount of water vapor lost through the skin was measured with a Tewameter® TM 300 (Courage-Khazaka electronic $\mathrm{GmbH}$, Cologne, Germany). The stratum corneum hydration $(\mathrm{SCH})$ was measured using a Corneometer ${ }^{\circledR}$ CM 825 (Courage-Khazaka electronic GmbH, Cologne, Germany). All measurements were carried out according to published guidelines.

\section{Statistical Analysis}

Results are reported as the mean of at least three replicates \pm standard deviation (SD). Statistical analysis regarding the permeation study was assessed by ANOVA, followed by Kruskal-Wallis test. Results from skin tolerance were analyzed by ANOVA, followed by t-student test. A p value below 0.05 was considered significant. Prism®, V. 5 software (GraphPad Software Inc., San Diego, CA, USA) was used for all statistical calculations.

\section{RESULTS}

\section{Characterization}

The obtained HGs were transparent. KT-C940-HG was highly viscous with limited flowability upon inverting the tube. KT-P407-HG was liquid at room temperature and gelled at skin temperature. No changes were observed in formulations after 3 months storage at room temperature $\left(25 \pm 2{ }^{\circ} \mathrm{C}\right)$.

Both formulations exhibited neutral or near neutral $\mathrm{pH}$ values: $7.2 \pm 0.2$ and $6.7 \pm 0.2$ at room temperature, and $6.7 \pm 0.1$ and $6.2 \pm 0.1$ at $32^{\circ} \mathrm{C}$ for KT-P407-HG and KTC940-HG, respectively. No statistical significant changes were observed over time ( $\mathrm{p}>0.05$ ). The drug content was found to be $99 \pm 0.5 \%$. Results of drug content uniformity test for both HGs indicated that the drug was properly and uniformly dispersed.

The swelling process of KT-C940-HG and KT-P407-HG followed a first order model (Fickian kinetic), which was represented by the kinetic constants $\mathrm{k}=0.5188 \mathrm{~h}-1$ and $0.29 \mathrm{~min}-1$, respectively (the plot is provided in the supplementary material). The PBS uptake rate was inversely proportional to the PBS in the HG. Initially, there was a fast PBS uptake, probably due to the dehydration of the HG, and then the rate of PBS uptake reached a plateau corresponding to the maximum SR in the case of KT-C940-HG (Qmax = 25.26). KT-P407-HG after a fast PBS uptake $($ Qmax $=1.15)$ the HG solubilized.

The KT-C940-HG was completely degraded in $24 \mathrm{~h}$. The degradation process followed a zero order kinetic ( $\mathrm{r} 2=$ 0.9987). The degradation process did not depend on the concentration of the remaining polymer. This process was represented by a kinetic constant $\mathrm{k}=4.36 \% / \mathrm{h}$. Nevertheless the degradation process of KT-P407-HG took place at a much faster pace. It was finished in $17 \mathrm{~min}$, and followed a first-order kinetic model ( $\mathrm{r} 2=0.9985)$ (graph is provided in the supplementary material).

The P result of KT-C940-HG was higher than KT-P407- HG, $98.70 \pm 3.76 \%$ and $74.93 \pm 7.96 \%$, respectively. HGs were examined by SEM. Fig. 1 shows the SEM micrographs of KT-C940-HG and KT-P407-HG. KT-C940HG exhibited three-dimensional heterogeneous filament structure with capillary channels interconnected (Fig. 1A and B), quite different from that of KT-P940-HG (Fig. 1C and D), in which is showed a porous structure more compact.

\section{Rheological Behavior}

Figure 2 shows the rheological behaviors of KT-C940-HG and KT-P407-HG at 25 and $32^{\circ} \mathrm{C}$. No changes on rheological behavior were observed for KT-C940-HG between 25 and $32^{\circ} \mathrm{C}$ although a slight decrease on viscosity 
was observed as the temperature increased, from $6.079 \pm 0.037$ to $5.057 \pm 0.066 \mathrm{~Pa} \cdot \mathrm{s}(\mathrm{p}<0.05)$. Herschel-Bulkley model was the model that statistically best fitted rheological behaviour in ascending and descending stretches at $25^{\circ} \mathrm{C}(\mathrm{r}=0.9999)$ and $32^{\circ} \mathrm{C}(\mathrm{r} 2=0.9998)$ in KTC940-HG in line with others studies (24). KT-C940- HG also exhibited apparent thixotropy as the flow curves displayed hysteresis loops with the downward streches below the upward streches (Fig. 2A and B). Conversely, KT-P407-HG showed a Newtonian flow at $25^{\circ} \mathrm{C}$ and the Newton equation fitted the experimental data perfectly $(\mathrm{r}=1)$ confirming that viscosity is not affected by changes in shear rate (Fig. 2C). However, at $32^{\circ} \mathrm{C}$ the Newtonian behavior turned to pseudoplastic, being the Cross equation the mathematical model that best fitted experimental data $(\mathrm{r} 2=0.9988)$ in ascending and descending stretches. The viscosity values (at $100 \mathrm{~s}-1$ ) were of $0.132 \pm 0.007 \mathrm{~Pa} \cdot \mathrm{s}$ at at $25^{\circ} \mathrm{C}$ and $0.876 \pm 0.008$ at $32^{\circ} \mathrm{C}$. Finally, the gelation temperature of $\mathrm{KT}-\mathrm{P} 407-\mathrm{HG}$ was recorded to be $31^{\circ} \mathrm{C}$.

\section{In Vitro Release}

As depicted in Fig. 3, KT was released from C940-HG and P407-HG in the first $2 \mathrm{~h}$ in a similar profile. This could be interpreted by viscosity of HGs formulations. The drug release pattern was rapid; reaching KT cumulative release values of $93.33 \pm 2.66 \%$ from $\mathrm{C} 940-\mathrm{HG}$ and $94.06 \pm 3.58 \%$ from $\mathrm{P} 407-\mathrm{HG}$. On the base of the smaller AIC value, the firstorder kinetic equation was the model that best fitted experimental data (Eq. 11) in which K values were $3.46 \pm 0.84 \mathrm{~h}-1$ and $1.37 \pm 0.22 \mathrm{~h}-1$ for KT-C940-HG and KT-P407-HG, respectively.

\section{Permeation Parameters}

Figure 4 shows the cumulative amounts of KT permeated from C940-HG and P407-HG. Table I shows the permeation parameters of KT calculated from the amounts permeated across the skin and the retained amount after 24 $\mathrm{h}$. The median values of the cumulative amount of KT permeated after $24 \mathrm{~h}$ were $20.25 \mu \mathrm{g}$ and $105.06 \mu \mathrm{g}$ for KTC940-HG and KT-P407-HG, respectively. The TL is the time of KT to permeate through the skin membrane and to diffuse into the receptor medium when the steady state of diffusion is reached. TL for KT-C940-HG was found to be $8.5 \mathrm{~h}$ and $4.9 \mathrm{~h}$ for KT-P407-HG. KT-C940-HG exhibited median Kp values of $0.93 \times 10-5 \mathrm{~cm} / \mathrm{h}$ and $4.23 \times 10-5 \mathrm{~cm} / \mathrm{h}$ for KT-P407-HG. The amounts of KT remaining in the skin are also depicted in Table I. Building on this data, other theoretical calculations could be estimated, such as Css, which is a useful prediction of plasma levels that would be achieved in humans after topical application of developed HGs, considering a human plasma clearance of $34.3 \mathrm{~mL} / \mathrm{min}(25)$, and $1 \mathrm{~cm} 2$ application area. Css of 0.058 and $0.241 \mu \mathrm{g} / \mathrm{mL}$ for KT-C940HG and KT- P407- HG. A non-parametric Mann-Whitney U test showed significant statistical differences (p < 0.05) for TL, J, Kp and Css between KT-C940-HG and KT- P407-HG.

\section{Percutaneous Absorption}

Table II shows the obtained results of KT recovered from the skin surface, SC, epidermis, and dermis after an exposure time of $24 \mathrm{~h}$. It could be observed that KT-C940-HG exhibited $1 \%$ of percutaneous absorption, whereas KT-P407-HG showed less than $0.4 \%$. The vast majority of KT remained on the surface of the skin in both formulations, $\sim 92 \%$ of the applied dose in the donor compartment. The highest concentration of KT from C940-HG following the surface was found in the epidermis. In the case of P407-HG, the highest concentration of KT following the surface was found in the $\mathrm{SC}$, at a concentration lower than $1 \%$. In contrast to P407-HG, residual amounts of KT were found in the receptor medium from C940-HG.

\section{Anti-Inflammatory Efficacy}

The anti-inflammatory activity test was examined using the acute ear skin edema model induced by topical application with TPA. A KT solution in acetone was taken as the reference of inflammatory inhibition. As shown in Fig. 5, the KT-P407-HG showed an ostensible reduction of inflammation of $15.4 \%$ compared to the reference. However, the KTC940-HG exhibited lower anti-inflammatory effect in comparison with the reference.

\section{Histological Studies}

Figure 6 shows the histological photomicrographs of treated tissues, as well as, positive and negative controls. The inflammation caused by TPA in mice ear (positive control) resulted in the presence of immune system cells in blood vessels (small points in purple showed in magnifications, Fig. 6B) as a response to the inflammation. No cells from the immune system were observed in the negative control of inflammation (Fig. 6A) or in samples from 
treated ears with KT-P407-HG (Fig. 6C). Finally, samples from KT-C940-HG showed small number of immune system cells in blood vessels (Fig. 6D).

\section{Cutaneous Experimental Parameters}

The application of tested HGs did not induce any visual skin irritation and were well tolerated. Figure 7 depicts the measurements of biomechanical parameters of skin after formulation application. As can be observed, no changes on skin temperature were observed for none of the HGs. In particular, the KT-P407-HG forms a visible film on the skin; at time of application KT-P407-HG is liquid and transparent but it gels a few minutes after its application onto the skin due to its gelation nature depending on the temperature. One hour after application, water has completely evaporated and a thin and transparent film can be seen to touch on the skin. Slight decrease in TEWL values for both HGs was recorded, although no statistically significant differences were found when compared with basal values. However, SCH suffered slight but statistically significant decrease following KT C940HG application $(\mathrm{p}<0.05)$. This fall was even more pronounced for KT-P407-HG.

\section{Discussion}

P407 consisting of $70 \%$ (w/w) PEO units, was chosen because is a low toxicity excipient approved by U.S. Food and Drug Administration for different types of preparations and its attractive use as delivery system through the skin for local pain and inflammations (26). C940 was selected based on its excellent organoleptic characteristics and high viscosity at low concentration, as well as, its ability to retain water leading to the formation of a crosslinked polymeric network decreases the loss of moisture from the skin surface hindering water evaporation (24). Among physical characteristics for dermal formulations $\mathrm{pH}$ is an important factor to avoid skin irritation or make the skin susceptible to bacterial infection (11). Natural acidity of the skin ranges from 4 to 6 , depending on the skin area and the age of the individual, due to the buffer system in the skin that is able to absorb small quantities of acid or alkali material applied to reduce irritation (27). Our $\mathrm{pH}$ findings make both formulations suitable for topical application. Although statistical differences between 25 and $32^{\circ} \mathrm{C}$ were found in both formulations ( $\mathrm{p}<$ 0.05). There were not implications in terms of tolerance and stability from the physiologic and technological points of views.

KT-C940-HG exhibited slower swelling behavior than KT-P407-HG. In KT-C940-HG the SR reached an equilibrium state within $\sim 5 \mathrm{~h}$ (supplementary material). This may be due to C940 possess low flexibility and high crosslinking degree (28). In contrast, KT-P407-HG after the critical micelle concentration (CMC) of P407 is a key factor in its sol-gel state. At concentrations below the CMC P407 is soluble in water, in our KT-P407-HG the polymer was $18 \%$, and once poured in the swelling medium the concentration of P407 decreased rapidly below CMC, thus producing the swelling and solubilized in $\sim 19$ min.

Morphological characteristics might contribute to the permeability, drug release and degradability properties. Morphological studies revealed a more compact surface in KT- P407-HG. However, this structure could be due to the drying process during SEM pre-treatment of samples, because drying induces collapse of the porous structure within the material. In the case of a micellar HGs (such as P407) a potential adhesion of these small structures could be occurred.

On the other hand, rheology is a useful technique to characterize the structure strength of semisolid systems and predict their behavior for topical uses (29). Concretely, viscosity of vehicles may play an important role in controlling the release and skin permeation of drugs (30). Rheological measurements indicated how much a sample deforms or how fast it flows by means of stressing the sample. For the KT-C940-HG the Herschel-Bulkley equation provides a general model for plastic materials that are pseudoplastic substances which additionally feature a yield point. The fluid requires a certain minimum stress to initiate flowing and after that it exhibits a pseudoplastic flow where the viscosity changes depending on the shear rate: the more shear rate, the lower viscosity (Fig. 2A). This behaviour is often seen in polymer systems; the hydrogel forms a three-dimensional network under static conditions, but it flows when stressed surpassing the yield point because the polymer chains become floworiented; therefore, the viscosity decreases. This behaviour is very interesting in semi-solid formulations because there is a high viscosity under static conditions that increases the physical stability of the formulation. Yet, the formulation thins under strain, desirable property for topical application. Furthermore, the apparent thixotropy exhibited evidenced a time-dependant shear thinning phenomenon when the system is stressed. This fact eases the spreading of the HG in a thin and homogeneous layer when it will be applied to the skin. 
KT-P407-HG fitted to Newton equation. This is consistent with the poloxamer under temperature value below solgel transition temperature, in which PEO blocks are hydrated, and PPO is relatively soluble in water showing low viscosity. In this case, the formulation was found to be easily spreadable and nondripping in nature. Indeed, the Newtonian behavior of the KT-P407-HG at low temperatures makes it suitable for application as a spray or roll-on. A reversible sol-gelstatetransition allows a cool solution to flow onto skin providing a non-occlusive gel at body temperature (31).

As reported previously, $\mathrm{P} 407-\mathrm{HG}$ exhibited reversible solgel transition behavior and becomes a gel above the critical gelation temperature (11). This critical gelation temperature was $31^{\circ} \mathrm{C}$, and thus gelled under release experiment conditions $\left(32^{\circ} \mathrm{C}\right)$, which might explain that release profiles of both formulations were similar. The KT release from HGs was mainly via diffusion. In the first-order kinetic model the release constant is independent of the initial drug concentration, and the release rate at any given time is directly proportional to the drug concentration in the matrix. The obtained $\mathrm{K}$ values were indicative that formulations could allow providing a great amount of drug quickly on the surface of SC, and enhancing drug absorption through skin (2).

The first ex vivo skin permeation study of KT from C940- HG and P407-HG was performed in order to determine the permeation profile of drug from these vehicles as well as skin permeation parameters. Ear pig skin was used to this experiment because is a representative membrane for percutaneous absorption due to its structural similarity with human skin and density of hair follicles (20). The most remarkable was that theoretical Css values demonstrated that were below the therapeutic plasma concentration $(0.3-5 \mu \mathrm{g} / \mathrm{mL})(25)$. This is a distinctive attribute in managing local symptoms, while minimizing the potential risk of systemic side-effects.

Topical administration at the site of the inflammation is usually the most effective route because a much higher concentration of the drug can be attained. The percutaneous absorption profile permits the evaluation of the margin of safety of a given compound that is incorporated in a designed topical formulation designed. It is known that the SC is the primary barrier against drug penetration therefore, the amount of KT absorbed percutaneously is assumed to be that from the epidermis, the dermis and the amount in the receptor medium.

From results obtained in KT skin distribution studies, it becomes apparent that the SC is the predominant barrier to KT penetration through the skin. It seems that C940-HG facilitated KT diffusion to the next layer of skin, the epidermis, in which the drug remained more retained. This finding was not surprising due to the highly hydrophilic nature of KT; both, the drug and the vehicle are hydrophilic compounds. Hence, it is expected that the drug remained bounded to the vehicle instead of diffusing through the skin. It might be a low vehicle-skin partition coefficient owing to the physicalchemistry properties of the drug and the vehicle. It is remarkable that for topical delivery systems, accumulation in the skin with minimal permeation is desired, in our case, antiinflammatory drug, this is an important premise for a local inflammations at low doses (32).

The relevant anti-inflammatory efficacy of KT-P407-HG revealed that KT-P407-HG applied on the mouse ear possesses enough ability to provide sufficient bioavailability KT to reach easily the site of action. Although no differences in KT skin accumulation were found between formulations when HGs were added to the human skin in the percutaneous absorption experiment, under in vivo conditions, the topical antiinflammatory efficiency of KT-P407-HG revealed a higher anti-inflammatory efficacy than KT-C940-HG. These differences observed between ex vivo and in vivo experiments may be explained by the absence of blood flow and metabolism ex vivo (4). Furthermore, the viable skin acts as a local reservoir filled with the carrier-associated drug for in vivo conditions. This reservoir is partially identical to the site of the desired biological action (33).

The inflammation process attract immune system cell to the affected tissue, blood vessels expand and become more porous allowing cells to leave the circulatory system and enter the damaged tissue. The histological results confirmed the in vivo anti-inflammatory efficacy showing that KT-P407-HG resulted in a more reduction of inflammation than KT-C940- HG.

In order to assess potential changes due to the formulation, variations in the skins properties were studied by biometrological techniques. TEWL measurement due to passive diffusion of water through the skin gives important information about skin barrier function (34). The $\mathrm{SCH}$ determined as conductance that the free water provides to the skin surface (35).

The skin barrier function is known to reside in the SC. P407 is widely used in medicine and biotechnology as biocompatible surfactant. Thus based on the higher antiinflammatory efficacy results of KT-P407-HG let us hypostatize a higher permeation through skin by modification of SC by decreasing lipid order (36-38). It could explain the slight change in TEWL (Fig. 6B), which was evident in SCH values (Fig. 6C). Besides, HGs are formed by 
water absorption; the polymer absorbs water and swells forming a threedimensional net structure. Therefore, the decrease of SCH for KT-C940-HG could be due to the capture of water from the skin surface.

\section{CONCLUSIONS}

Two HG systems loading KT have been elaborated for the local treatment of inflammation. These formulations were stable HGs with suitable physical properties for topical application. The in vitro release studies demonstrated a fast release profile of KT. Although KT-P407-HG presented higher permeated and retained amounts in the skin, no theoretical systemic plasma concentrations were predicted to be reached. However, the skin layer distribution of KT showed that C940-HG diffused to deeper layers of the human skin. KTP407-HG was the formulation with optimal antiinflammatory efficacy. Finally, formulations did not cause any irritant effects, and were well tolerated. For these reason KT-P407-HG was proposed as suitable formulation for antiinflammatory local treatment without theoretical systemic side effect. However, additional in vivo studies should be addressed assessing the in vivo bioavailability of KT after dermal treatment of our formulation.

\section{ACKNOWLEDGMENTS AND DISCLOSURES}

This work was supported by the Ministry of Science and Innovation of Spain for the financial (MAT201459134R). Prof. Juan Blasi and Inmaculada Gómez de Aranda from the Department of Pathology and Experimental Therapeutics are also acknowledged for their excellent technical support in histological studies. The authors report no conflict of interests, financial or otherwise. All procedures performed in studies involving human participants were in accordance with the ethical standards of the institutional and/or national research committee and with the 1964 Helsinki declaration and its later amendments or comparable ethical standards. Informed consent was obtained from all individual participants included in the study. All procedures performed in studies involving animals were in accordance with the ethical standards of the institution or practice at which the studies were conducted.

\section{REFERENCES}

1.De Oliveira Jr GS, Agarwal D, Benzon HT. Perioperative single dose ketorolac to prevent postoperative pain: ameta-analysis of randomized trials. Anesth Analg. 2012;114(2):424-33

2. El-Setouhy DA, El-Ashmony SM. Ketorolac trometamol topical formulations: release behaviour, physical characterization, skin permeation, efficacy and gastric safety. J Pharm Pharmacol. 2010;62(1):25-34.

3.Ting WW, Vest CD, Sontheimer RD. Review of traditional and novel modalities that enhance the permeability of local therapeutics across the stratum corneum. Int J Dermatol. 2004;43(7):538-47.

4. Vega E, Egea MA, Garduño-Ramírez ML, García ML, Sánchez E, Espina M, Calpena AC. Flurbiprofen PLGA-PEG nanospheres: role of hydroxy- $\beta$-cyclodextrin on ex vivo human skin permeation and in vivo topical anti-inflammatory efficacy. Colloids Surf B: Biointerfaces. 2013;110:339-46.

5. Koop HS, de Freitas RA, de Souza MM, Savi-Jr R, Silveira JL. Topical curcumin-loaded hydrogels obtained using galactomannan from Schizolobium Parahybae and xanthan. Carbohydr Polym. 2015;116:229-36.

6. Guvendiren M, Lu HD, Burdick JA. Shear-thinning hydrogels for biomedical applications. Soft Matter. $2012 ; 8: 260 \mathrm{e} 272$.

7. Ahmed EM. Hydrogel: preparation, characterization, and applications: a review. J Adv Res. 2015;6(2):105-21.

8. Hoare TR, Kohane DS. Hydrogels in drug delivery: progress and challenges. Polymer. 2008;49:1993-2007.

9. Miguel SP, Ribeiro MP, Brancal H, Coutinho P, Correia IJ. Thermoresponsive chitosan-agarose hydrogel for skin regeneration. Carbohydr Polym. 2014;111:366-73. 
10. de Araújo DR, da Silva DC, Barbosa RM, Franz-Montan M, Cereda CM, Padula C, et al. Strategies for delivering local anesthetics to the skin: focus on liposomes, solid lipid nanoparticles, hydrogels and patches. Expert Opin Drug Deliv. 2013;10(11): 1551-63.

Brugués AP, Naveros BC, Calpena Campmany AC, Pastor PH, Saladrigas RF, Lizandra CR. Developing cutaneous applications of paromomycin entrapped in stimuli-sensitive block copolymer nanogel dispersions. Nanomedicine (London). 2015;10(2):227-40.

Chawla V, Saraf SA. Rheological studies on solid lipid nanoparticle based carbopol gels of aceclofenac. Colloids Surf B: Biointerfaces. 2012;92:293-8.

13. Das B, Nayak AK, Nanda U. Topical gels of lidocaine $\mathrm{HCl}$ using cashew gum and Carbopol 940: preparation and in vitro skin permeation. Int J Biol Macromol. 2013;62:514-7.

14. ICH harmonised tripartite guideline. Validation of analytical procedures: text and methodology Q2(R1). Geneva: ICH; 2005.

15. Guidance document for the conduct of skin absorption studies. OECD series on testing and assessment No.28. Paris: OECD; 2004.

16. Skin absorption: in vitro Method. OECD guideline for the testing of chemicals. Guideline 428.Paris: OECD; 2004.

17. Basic criteria for the in vitro assessment of dermal absorption of cosmetic ingredients. SCCS/1358. Brussels: Scientific Committee on Consumer Safety; 2010.

18. Williams AC. Transdermal and topical drug delivery. London: Pharmaceutical Press; 2003.

19. Flo A, Calpena AC, Halbaut L, Araya EI, Fernández F, Clares B. Melatonin delivery: transdermal and transbuccal evaluation in different vehicles. Pharm Res. 2016;33(7):1615-27.

20. Alonso C, Lucas R, Barba C, Marti M, Rubio L, Comelles F, et al. Skin delivery of antioxidant surfactants based on gallic acid and hydroxytyrosol. J Pharm Pharmacol. 2015;67(7):900-8.

21. Domínguez-Villegas V, Clares-Naveros B, García-López ML, Calpena-Campmany AC, BustosZagal P, Garduño-Ramírez ML. Development and characterization of two nano-structured systems for topical application of flavanones isolated from Eysenhardtia Platycarpa. Colloids Surf B: Biointerfaces. 2014;116:183-92.

22. World medical association. World medical association declaration of Helsinki: ethical principles for medical research involving human subjects. JAMA. 2013;310(20):2191-4.

23. Clarys P, Clijsen R, Taeymans J, Barel AO. Hydration measurements of the stratum corneum: comparison between the capacitance method (digital version of the Corneometer CM 825(R)) and the impedance method (Skicon-200EX(R)). Skin Res Technol. 2012;18(3):316-23.

24. Lee SG, Kim SR, Cho HI, Kang MH, Yeom DW, Lee SH, Lee S, Choi YW. Hydrogel-based ultramoisturizing cream formulation for skin hydration and enhanced dermal drug delivery. Biol Pharm Bull. 2014;37(10):1674-82.

25. Cordero JA, Alarcon L, Escribano E, Obach R, Domenech J. A comparative study of the transdermal penetration of a series of nonsteroidal antiinflammatory drugs. J Pharm Sci. 1997;86(4): 503-8.

26. Escobar-Chávez JJ, López-Cervantes M, Naïk A, Kalia YN, Quintanar-Guerrero D, GanemQuintanar A. Applications of thermo-reversible pluronic F-127 gels in pharmaceutical formulations. J Pharm Pharm Sci. 2006;9(3):339-58.

27. Sierra AF, Ramirez ML, Campmany AC, Martinez AR, Naveros BC. In vivo and in vitro evaluation of the use of a newly developed melatonin loaded emulsion combined with UV filters as a protective agent against skin irradiation. J Dermatol Sci. 2013;69(3):202-14.

28. Tang C, Yin L, Pei Y, Zhang M, Wu L. New superporous hydrogels composites based on aqueous carbopol ${ }^{\circledR}$ solution (SPHCcs): synthesis, characterization and in vitro bioadhesive force studies. Eur Polym J. 2005;4(3):557-62. 
29. Dong L, Liu C, Cun D, Fang L. The effect of rheological behavior and microstructure of the emulgels on the release and permeation profiles of Terpinen-4-ol. Eur J Pharm Sci. 2015;78:14050 .

30. Dewan M, Bhowmick B, Sarkar G, Rana D, Bain MK, Bhowmik M, et al. Effect of methyl cellulose on gelation behavior and drug release from poloxamer based ophthalmic formulations. Int J Biol Macromol. 2015;72:706-10.

31. Dumortier G, Grossiord JL, Agnely F, Chaumeil JC. A review of poloxamer 407 pharmaceutical and pharmacological characteristics. Pharm Res. 2006;23(12):2709-28.

32. Almeida H, Amaral MH, Lobão P, Lobo JM. Pluronic F-127 and pluronic lecithin organogel (PLO): main features and their applications in topical and transdermal administration of drugs. J Pharm Pharm Sci. 2012;15(4):592-605.

33. Cevc G, Blume G. Biological activity and characteristics of triamcinolone-acetonide formulated with the self-regulating drug carriers. Transfersomes Biochim Biophys Acta. 2003;1614(2): 15664.

34. Mohammed D, Hirata K, Hadgraft J, Lane ME. Influence of skin penetration enhancers on skin barrier function and skin protease activity. Eur J Pharm Sci. 2014;51:118-22.

35. del Pozo A, Viscasillas A. Efficacy evaluation. In: Salvador A, Chisvert A, editors. Analysis of cosmetic products. Amsterdam: Elsevier; 2007. p. 462-74.

36. Shin SC, Cho CW, Oh IJ. Effects of non-ionic surfactants as permeation enhancers towards piroxicam from the poloxamer gel through rat skins. Int J Pharm 2001;222(2):199-203.

37. Erukova VY, Krylova 00, Antonenko YN, Melik-Nubarov NS. Effect of ethylene oxide and propylene oxide block copolymers on the permeability of bilayer lipid membranes to small solutes including doxorubicin. Biochim Biophys Acta. 2000;1468(1-2):73-86.

38. Demina T, Grozdova I, Krylova O, Zhirnov A, Istratov V, Frey H, et al. Relationship between the structure of amphiphilic copolymers and their ability to disturb lipid bilayers. Biochemistry. 2005;44(10): 4042-54. 
Figures and Tables

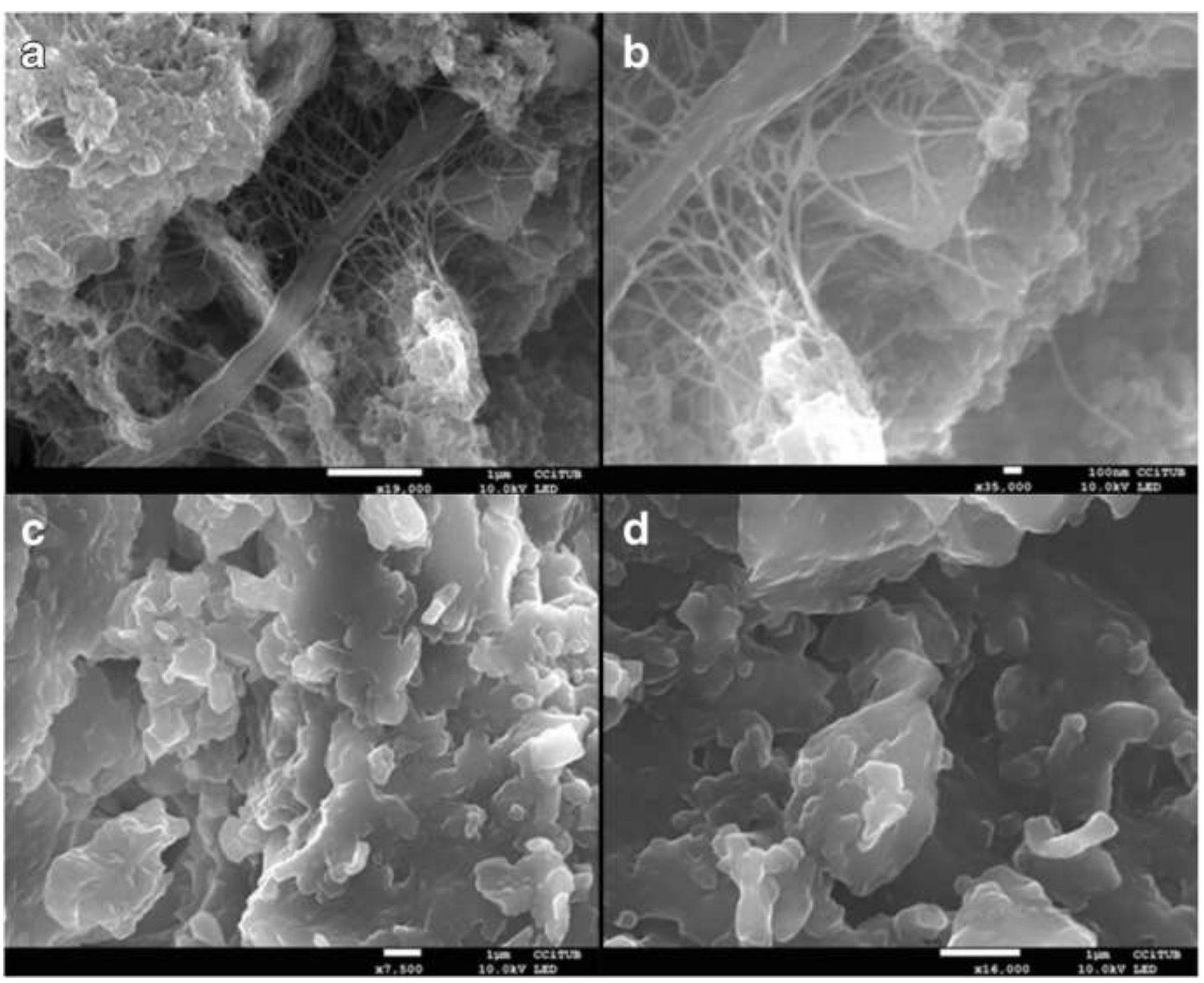

Fig. 1 SEM images of dried discs of (A) KT-C940-HG (x19000); (B) KT-C940-HG (x35000); (C) KTP407-HG (x7500) and (D) KTP407-HG (x16000) 
a
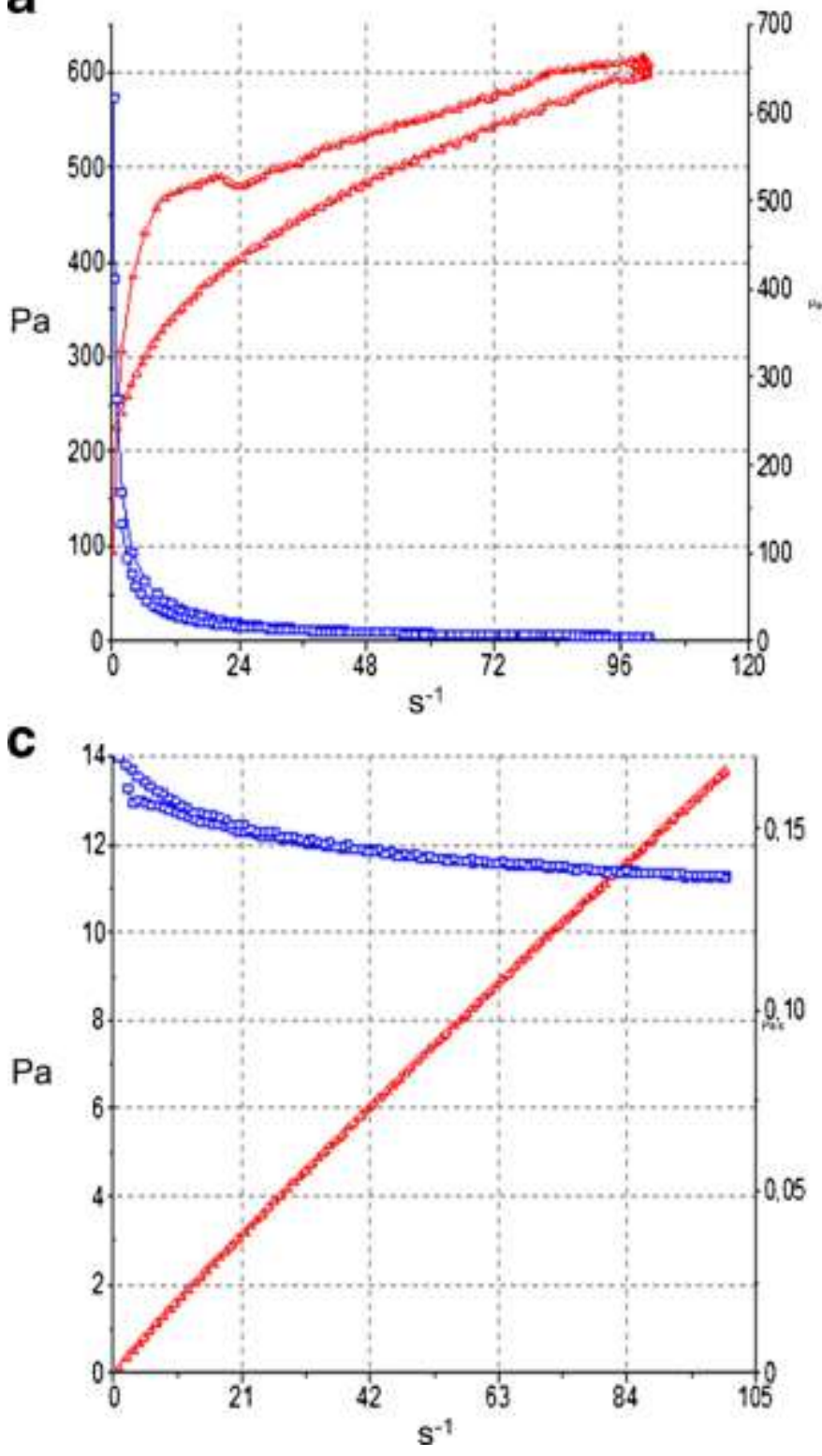

b

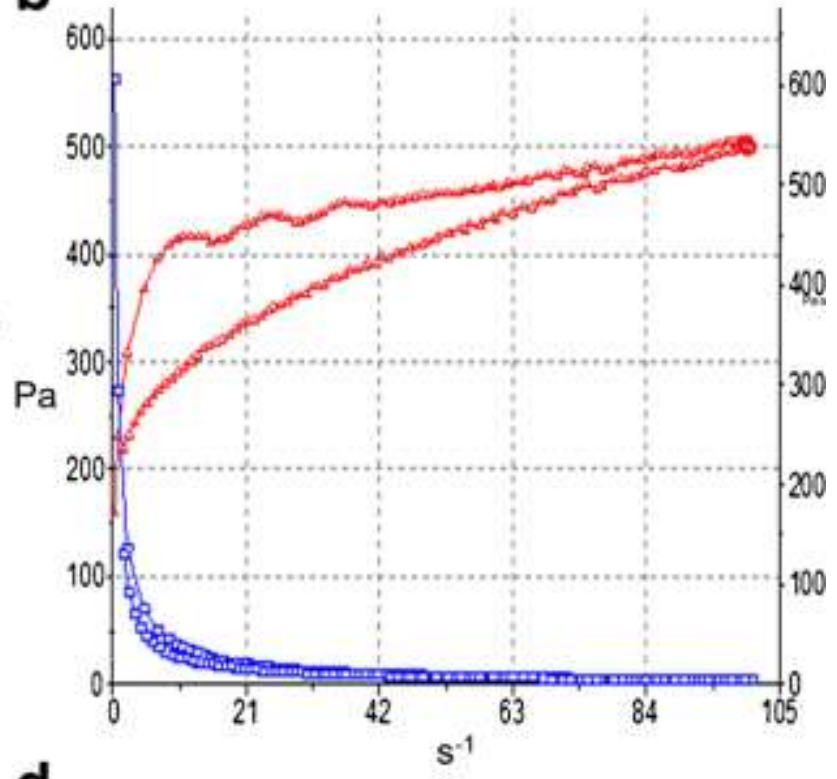

d

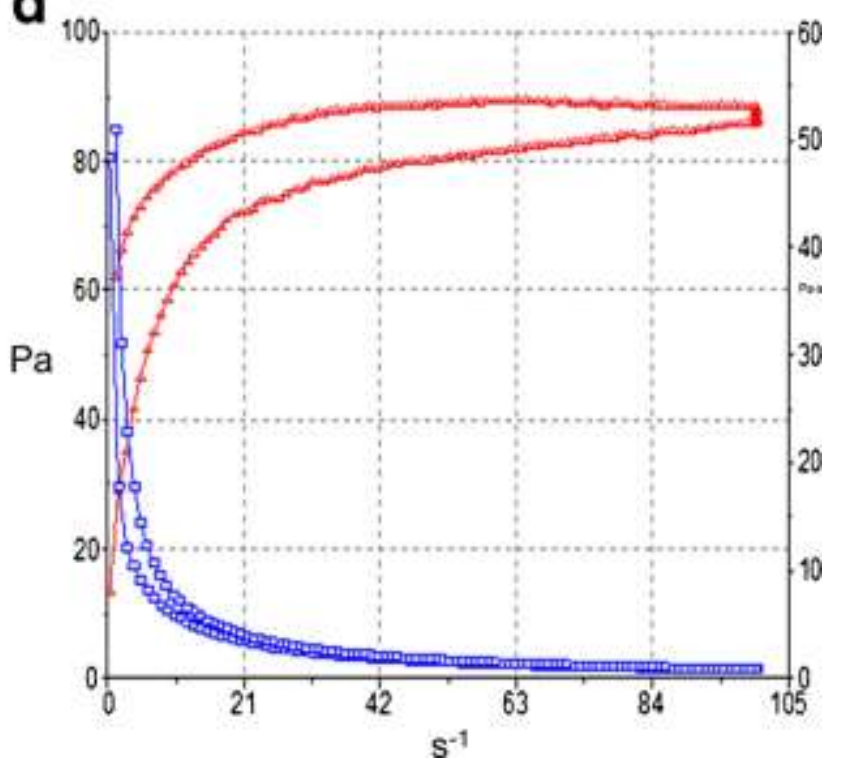

Fig. 2 Rheograms of the two tested formulations: (A) KT-C940-HG at $25^{\circ} \mathrm{C}$; (B) KT-C940-HG at $32^{\circ} \mathrm{C}$; (C) KT-P407-HG at $25^{\circ} \mathrm{C}$; (D) KT-P407-HG at $32^{\circ} \mathrm{C}$. The flow curve represents shear stress (Pa) versus shear rate $(\mathrm{s}-1)$ in red, and the viscosity curve represents viscosity $(\mathrm{Pa} \cdot \mathrm{s})$ versus shear rate $(\mathrm{s}-1)$ in blue. 


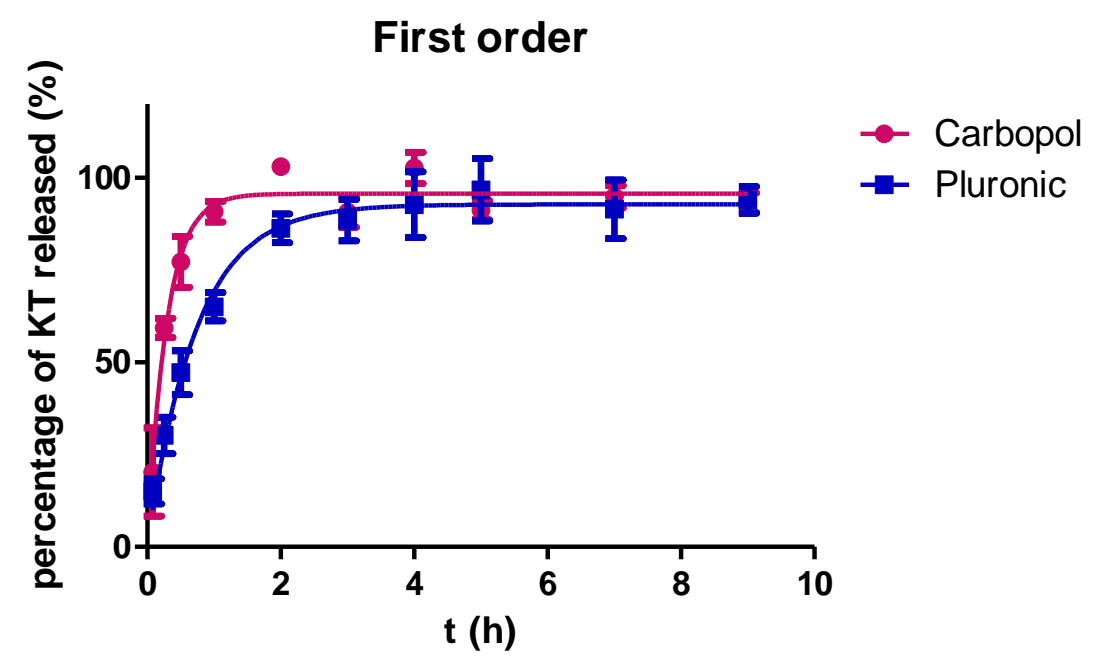

Fig. 3 Cumulative amount of KTreleased from formulations for $9 \mathrm{~h}$. Data at each time point represent mean $\pm \mathrm{SD}$ of at least 4 experiments. 


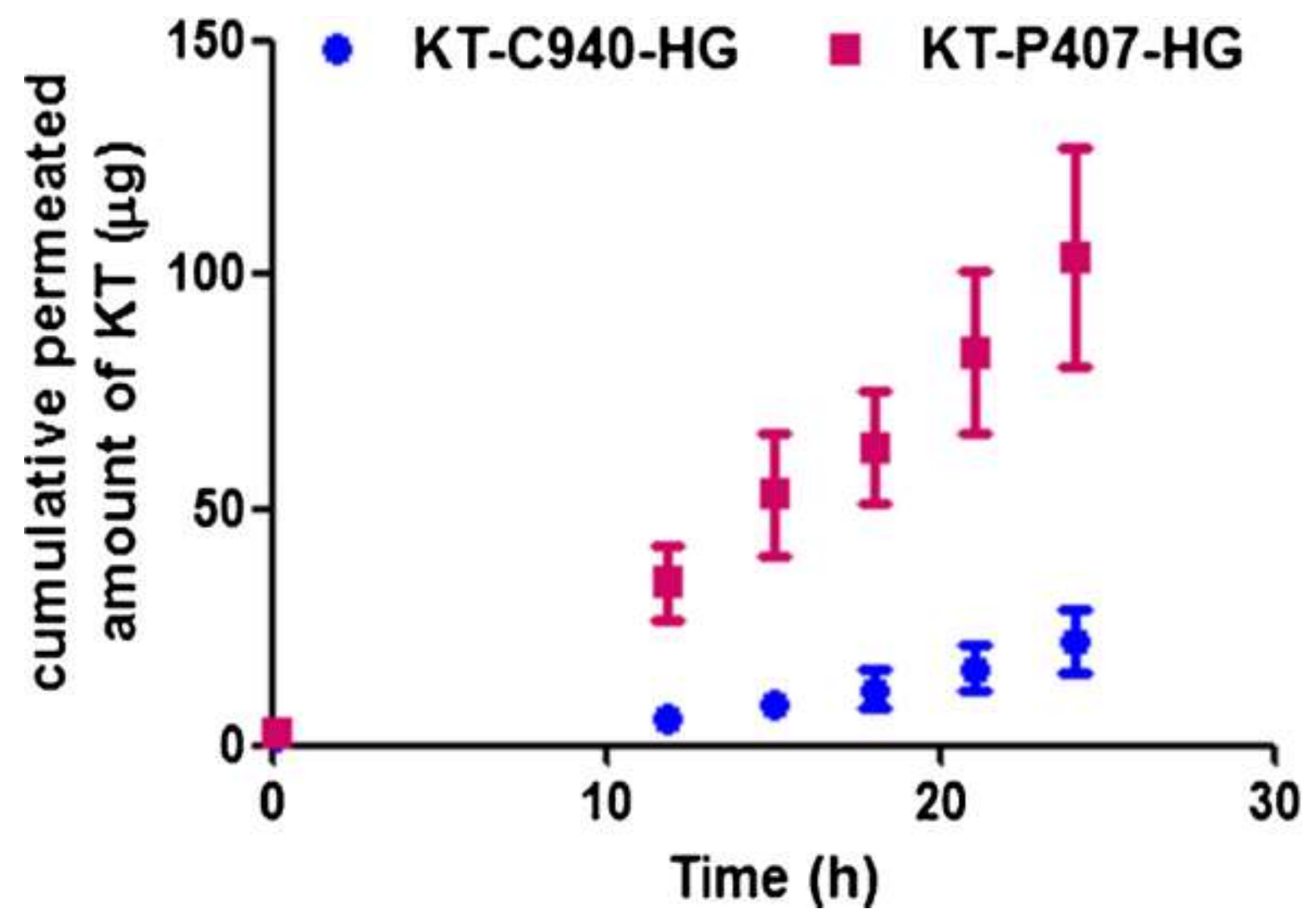

Fig. 4 Cumulative amount of KT permeated through porcine ear skin from formulations for $24 \mathrm{~h}$. Data at each time point represent mean $\pm \mathrm{SD}$ of at least 6 experiments. 


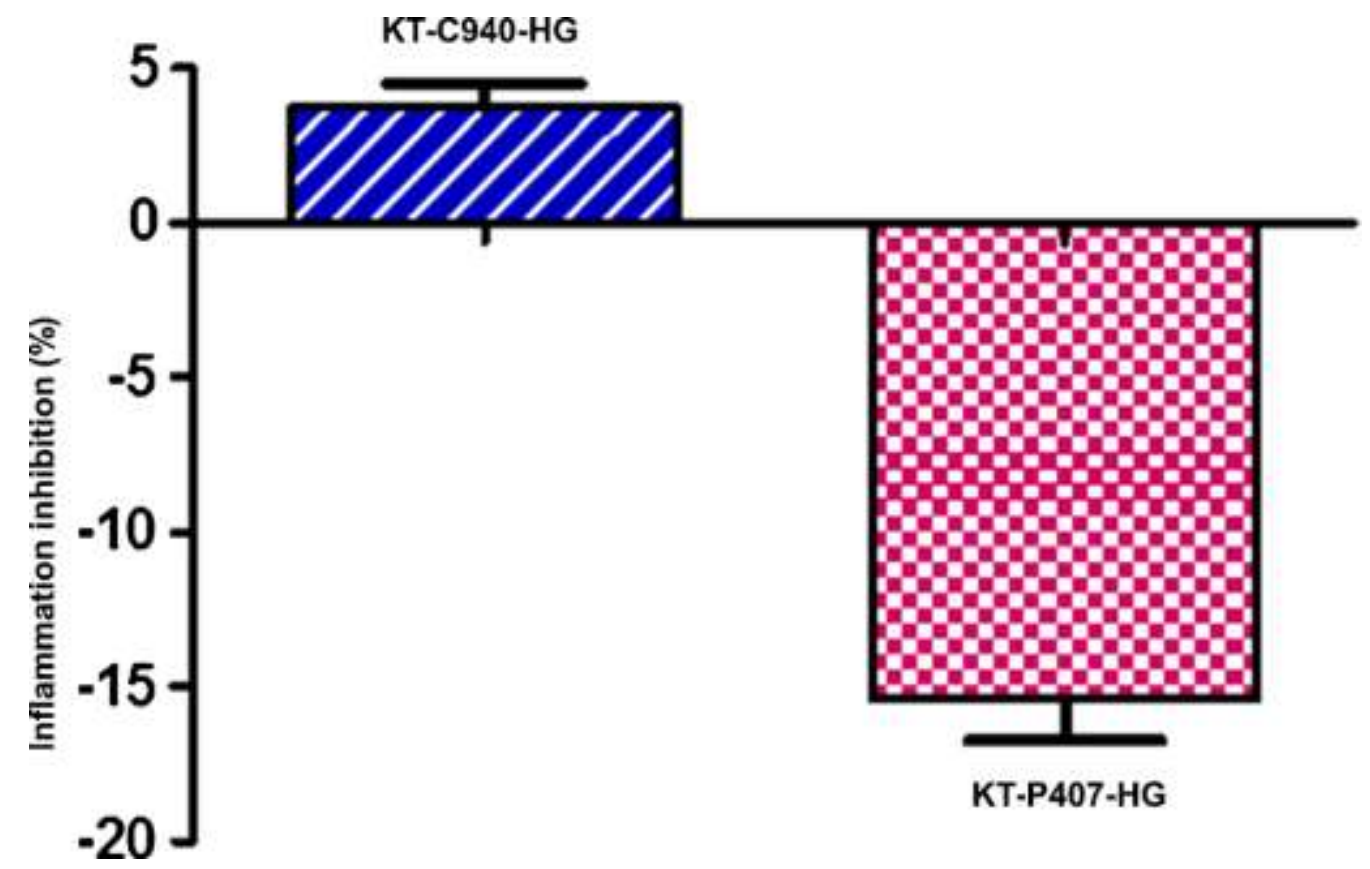

Fig. 5. Anti-inflammatory efficacy of tested formulations as the percentage of reduction of inflammation compared to the reference. Results are expressed as mean \pm SD of 3 determinations. 

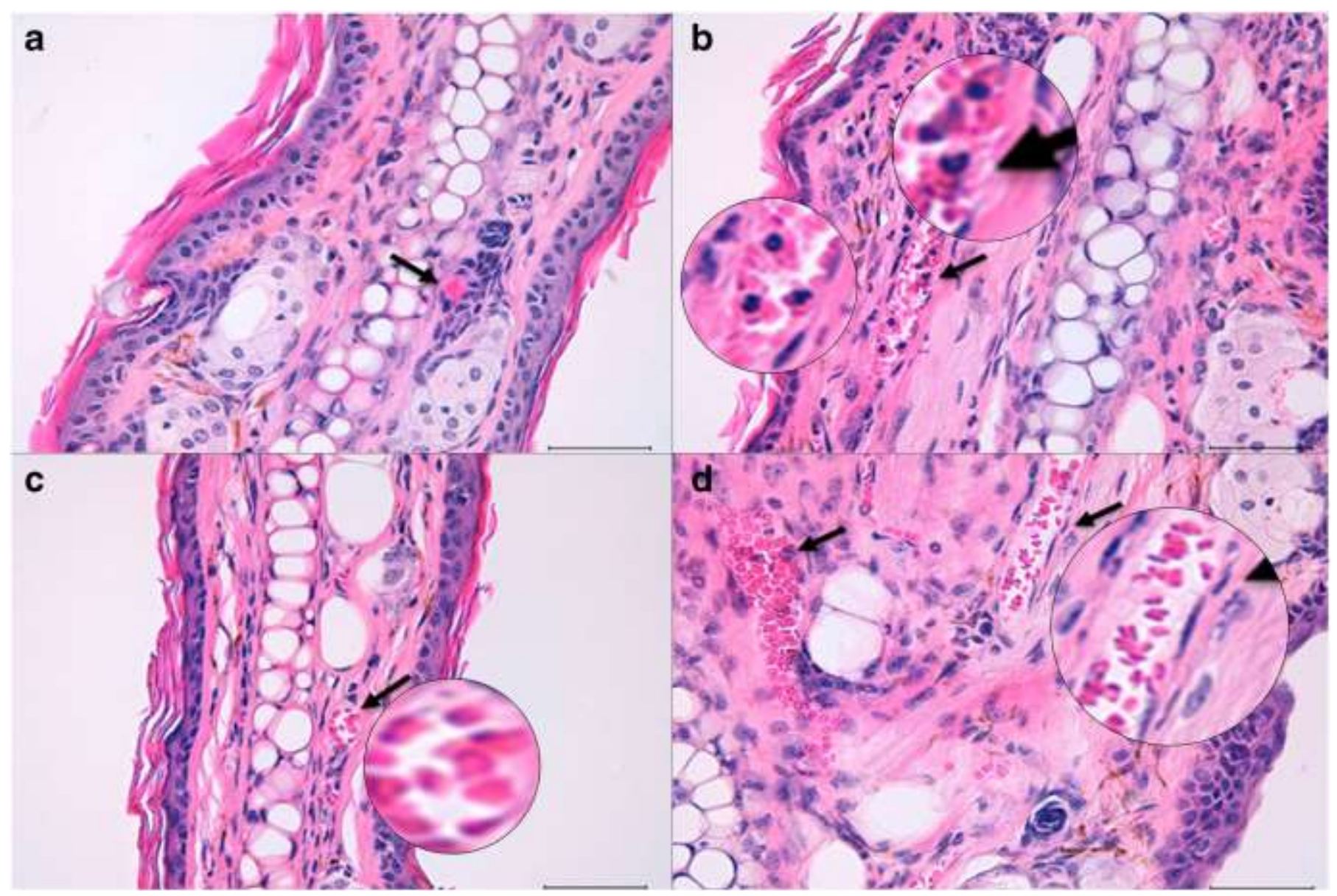

Fig. 6 Histological images of the mice ears. (A) negative control of inflammation; (B) positive control of inflammation; (C) inflamed ear treated with KT-P407- HG; (D) inflamed ear treated with KT-C940-HG. Bars length $50 \mu \mathrm{m}$. 

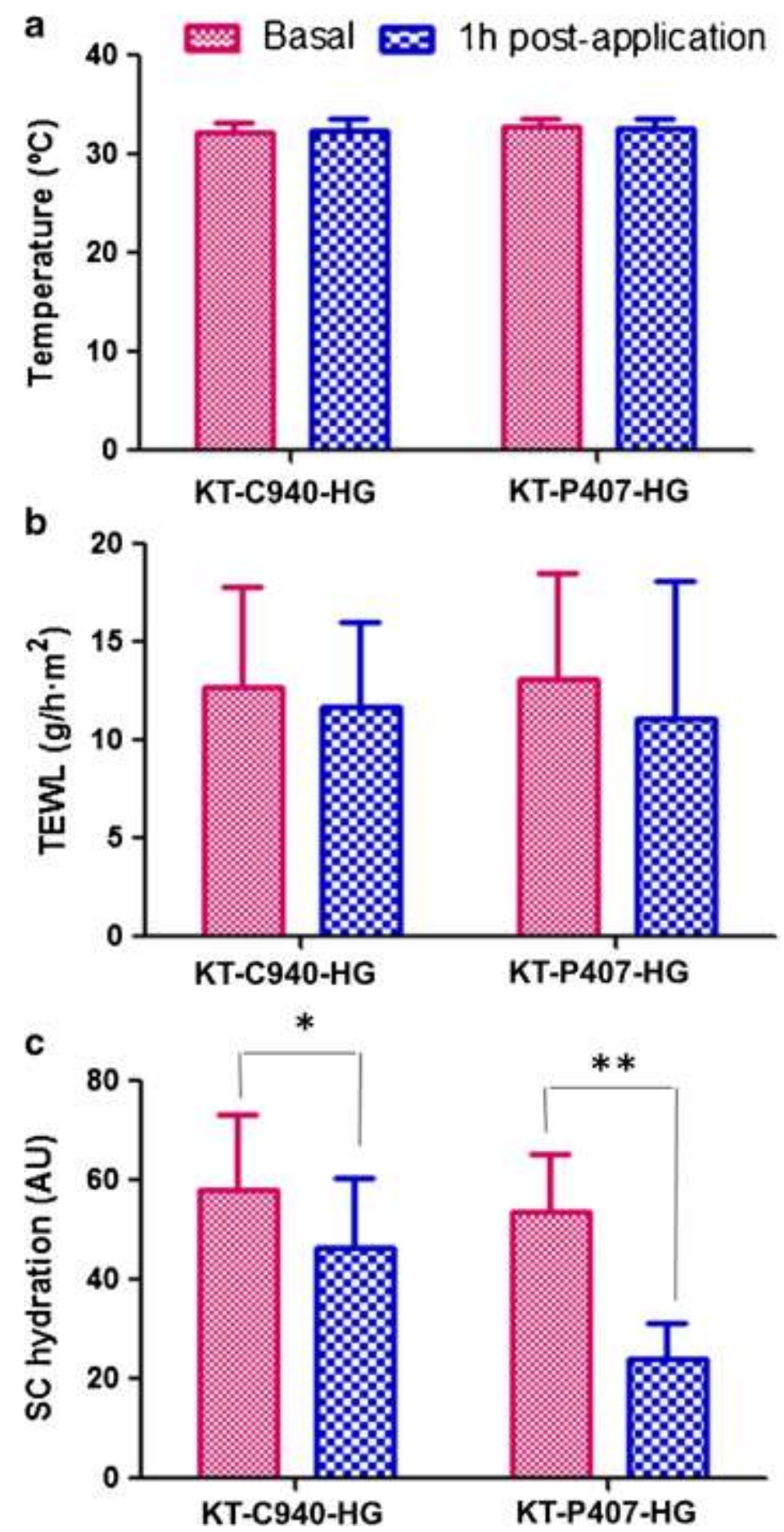

Fig. 7 Evolution of biomechanical parameters monitored before the application of the formulations and $1 \mathrm{~h}$ after application. Temperature is expressed as degrees Celsius $\left({ }^{\circ} \mathrm{C}\right)$, TEWL is expressed as $\mathrm{g} / \mathrm{h} \times \mathrm{cm} 2$, and the SCH as arbitrary units (AU) $\left(^{*}=\mathrm{p}<0.005\right.$; $\left.^{* *}=\mathrm{p}<0.0005\right)$. 
Table 1. Transdermal Permeation Parameters of KTafter 24 h. Results are Expressed as Median (Minimum-Maximum)

\begin{tabular}{lll} 
Parameters & KT-C940-HG & KT-P407-HG \\
\hline AP24h $(\mu \mathrm{g})$ & $20.25(17.68-26.81)$ & $105.06(87.83 .-120.29)$ \\
AR $(\mu \mathrm{g} / \mathrm{g}$ skin/cm2) & $192.01(188.88-347.94)$ & $1475.84(773.38-2107.53)$ \\
TL $(\mathrm{h})$ & $8.5(7.5-8.9)$ & $4.9(7.04-9.08)$ \\
$\mathrm{J}(\mu \mathrm{g} / \mathrm{cm} 2 / \mathrm{h})$ & $2.01(1.67-2.27)$ & $8.16(7.04-9.08)$ \\
$\mathrm{Kp}(\times 10-5)(\mathrm{cm} / \mathrm{h})$ & $0.93(0.83-1.14)$ & $4.23(3.52-4.54)$ \\
$\mathrm{Css}(\mu \mathrm{g} / \mathrm{mL})$ & $0.058(0.049-0.066)$ & $0.241(0.205-0.265)$ \\
\hline
\end{tabular}


Table 2. Distribution of KT within the skin from the three formulations after an exposure time of $24 \mathrm{~h}$. Results are expressed as mean values \pm standard deviations for 3 cells.

\begin{tabular}{lll} 
Percentage of KT & Carbopol ${ }^{\circledR}$ gel & Pluronic $®$ gel \\
\hline S & $92,89 \pm 5,04$ & $92,61 \pm 4,52$ \\
SC & $0,29 \pm 0,19$ & $0,27 \pm 0,19$ \\
E & $1,29 \pm 0,01$ & $0,12 \pm 0,07$ \\
D & b.l.q. & b.l.q \\
RF & $0,04 \pm 0,03$ & b.l.d \\
\hline Total recovery & $94,51 \pm 5,24$ & $93,01 \pm 4,37$ \\
\hline Percutaneous absorption & $1,34 \pm 0,01$ & $0,12 \pm 0,07$ \\
\hline b.l.q.: below limit quantification; b.l.d.: below limit detection
\end{tabular}

